\title{
Clinical and histopathological relationship of sildenafil and bosentan treatments in rats with monocrotaline induced pulmonary hypertension
}

\author{
Karpuz D ${ }^{1}$, Hallioglu O${ }^{1}$, Buyukakilli B², Gurgul S³, Balli E4, Ozeren $\mathrm{M}^{5}$, Tasdelen B ${ }^{6}$ \\ Department of Pediatrics, Division of Pediatric Cardiology, University of Mersin Faculty of Medicine, \\ Mersin, Turkey.drderyakarpuz@gmail.com
}

\begin{abstract}
BACKGROUND: Pulmonary arterial hypertension (PAH) is a challenging disorder characterized by increasing pulmonary artery pressure, which is hard to treat.

OBJECTIVE: This study was aimed to investigate the effects of bosentan, sildenafil and their combination. METHODS: Saline or MCT were applied to Wistar rats. By the development of PAH (4th week), MCT-given rats were treated orally with bosentan, sildenafil and combination of sildenafil and bosentan or placebo. ECHO examinations were performed. Tissues obtained from all of the rats were evaluated under an electron microscope. RESULTS: Left ventricular end diastolic diameter significantly increased in sildenafil and combined groups. Sildenafil group revealed a significant decrease in RV pressure and wall thickness. Examination of lung revealed a significant amount of connective tissue formation and increase in inflammatory cells in all the groups except controls in the interalveolar septum. Examination of PA revealed an increase in connective tissue volume, hypertrophic changes and expansions in granular endoplasmic reticulum cisternaes in smooth muscle cells in active groups rather than in the controls. Unlike the controls, the examination of the RV revealed an enlargement of the sarcoplasmic reticulum cisternaes in some cells, due to the calcium increase.

CONCLUSION: Sildenafil and the combined therapy demonstrated to have more impact on pressure and the RV parameters in rats, with lower inflammatory findings in lung tissue (Fig. 6, Ref. 31). Text in PDF www.elis.sk. KEY WORDS: bosentan, pulmonary hypertension, sildenafil,
\end{abstract}

\section{Introduction}

Pulmonary hypertension $(\mathrm{PH})$ is characterized by a progressive increase in pulmonary arterial pressure and pulmonary vascular resistance (PVR), which can result in right heart failure, re-modelling in lung vasculature and death (1-4). Right ventricular failure, which develops secondary to right ventricular remodelling, is the main prognostic factor that leads to death in patients with PAH (5). Medial hypertrophy in the distal pulmonary arteries, proliferative and fibrotic changes of intima, perivascular inflammatory infiltration, vasoconstriction, thrombosis, adventitial thickening and in the end the remodelling of pulmonary arteriae and increase in vascular resistance are the factors that play a role in change of

${ }^{1}$ Department of Pediatrics, Division of Pediatric Cardiology, University of Mersin Faculty of Medicine, Mersin, Turkey, ${ }^{2}$ Department of Biophysics, University of Mersin Faculty of Medicine, Mersin, Turkey, ${ }^{3}$ Department of Biophysics, University of Gaziosmanpasa Faculty of Medicine, Tokat, Turkey, ${ }^{4}$ Department of Histology and Embryology, University of Mersin Faculty of Medicine, Mersin, Turkey, ${ }^{5}$ Department of Cardiovascular Surgery, University of Mersin Faculty of Medicine, Mersin, Turkey, and ${ }^{6}$ Department of Biostatistics, University of Mersin Faculty of Medicine, Mersin, Turkey

Address for correspondence: D. Karpuz, MD, Department of Pediatrics, Division of Pediatric Cardiology, University of Mersin Faculty of Medicine, Mersin, Turkey.

Phone: +90.324.2410000, Fax: +90.324.2410098 histopathological architecture of $\mathrm{PH}$ (6). Although there are many causes in the pathogenesis of $\mathrm{PH}$, the most important issue is the progressive endothelial cellular dysfunction. Since PAH is a progressive disorder, early diagnosis and treatment is very important (6). Various pharmacologic agents have been used in pulmonary arterial hypertension, which are effective in improving quality of life, survival and symptomatic status of patients. Bosentan, an endothelin A and B (ETA/ETB) receptor antagonist (7-9), and sildenafil, a phosphodiesterase type 5 (PDE5) inhibitor, and the combination of these two drugs are the most effective treatment modalities used in PH (10). However, there are controversy discussions about the possibility of shortening the effective period in the long term uses. Clinical studies have been carried out on human subjects although histopathological studies could not be performed ethically. Thus, in order to understand the pathophysiological processes of PAH clearly and to compare the treatment modalities used in PAH, this can possibly be made by using experimental models (11). MCT, the most commonly used drug in experimental models to induce $\mathrm{PAH}$, is the agent which causes a direct endothelial damage that leads to $\mathrm{PH}$ (11) as well as medial hypertrophy and inflammatory response. This study was planned to study the effects of bosentan, sildenafil and combined therapy on ECHO parameters, histopathology of lung, right heart and pulmonary artery in rats, in which PAH is developed using MCT administration. 


\section{Methods}

\section{Animal model}

48 male 3 months of age Wistar rats weighting 200-300 g were included in the study and they were randomly divided into the five groups. All the groups except the control $(\mathrm{n}=8)$ were treated with MCT subcutaneously at a dose of $60 \mathrm{mg} / \mathrm{kg}$ (adjusted with $1 \mathrm{~N} \mathrm{HCl}$ dissolved and $\mathrm{pH} 7.4$ with $1 \mathrm{~N} \mathrm{NaOH}$ and 20 $\mathrm{mg} / \mathrm{ml})$. It has been reported that MCT in the above mentioned dosages cause PAH in both adult and infant rat models (12). At the end of four weeks following PAH development, one of the groups was treated with bosentan $(n=8)$, the other with sildenafil $(n=9)$ and the last one with the combined therapy bosentan and sildenafil $(\mathrm{n}=13)$. Treatment was not administered to one of the groups, in which PAH was developed $(n=10)$. Bosentan was administered at a dose of $300 \mathrm{mg} / \mathrm{kg} /$ day, while sildenafil was administered at a dose of $100 \mathrm{mg} / \mathrm{kg} /$ day as was suggested by Clozel et al (13).

Echocardiography measurements, tail pressures, weight and, clinical follow-up's were noted at the initiation of MCT administration, at the fourth week and seventh weeks following MCT administration. Rats were ventilated through the ventilator, when all the measurements were noted in order to measure main pulmonary artery pressures using invasive methods. Heart, pulmonary artery and lung tissue were evaluated using an electron microscopy. The pertinent acceptance needed was obtained from the Local Ethics Committee for Experimental Studies of Mersin University (02.06.2011, no: 31).

\section{Echocardiography}

Echocardiography was performed under a general anaesthesia while the rats were breathing spontaneously and laying on the back. Transthoracic two-dimensional (2D) echocardiography device (Vivid I S/N: 001651, General Electric, Tirat Convel, Israel), which has $\mathrm{M}$ mode and doppler options; the measurements were performed using $10 \mathrm{MHz}$ transducer. Right ventricular wall thickness (RVWT) was measured in 2D mode from parasternal short axe window, where the free wall could be seen best. Right ventricular end diastolic diameter (RVEDD) was measured from apical window, while the tricuspid valve was closed; the longest distance from the midpoint to right ventricular free wall and interventricular septum was noted.

Tricuspid valve insufficiency was used in order to assess right ventricular function and monitorization of PAH. Tricuspid valve insufficiency was assessed with a continuous-wave Doppler and tricuspid flow colour from apical window, where it could have been assessed best. Systolic right ventricular pressure was calculated using Bernoulli calculation $\left(\mathrm{p}=4 \mathrm{xTY}^{2}\right)$.

\section{Indirect blood pressure records}

Systolic and mean arterial blood pressure of rats were measured using the tail cuff method. Non-invasive indirect blood measurement system (MAY NIBP200-A, Commat LTD. ŞTI, Ankara , Turkey), which is compatible with BIOPACMP100 Acquisition System (Version 3.5.7, Santa Barbara, USA) was used. Five re- cords were taken for each rat. Systolic and the mean arterial blood pressure of rats was calculated by taking the average of the five records of each rat by using BIOPAC Acknowledge (ACK 100, version W5.7).

\section{Pulmonary artery pressure measurements}

Under a general anaesthesia with spontaneous respiration, trachea was dissected and fenestration of trachea was performed. 16 gauge cannula was placed into trachea through this window, fixed with $3 / 0$ silk suture and connected to mechanic ventilator (Harvard apparatus 55-7059 inspira ASV ventilator, MA USA). Peak pressure, respiration number/minute and $\mathrm{FiO} 2$ were adjusted as $10 \pm 2 \mathrm{cmH}_{2} \mathrm{O}, 60 \pm 5 / \mathrm{min}$ and $100 \%$, respectively. Then, mediastinum was opened following sternotomy and pulmonary artery was isolated. Pulmonary Artery was cannulated using a 26 gauge cannula. Pulmonary artery pressure was monitored using a pressure transducer (GTA 303 General transducer amplifier biopac systems MP150, Inc, USA) and a software system. After all the pressure recordings were obtained, the heart together with pulmonary artery and aorta was resected, than, right middle lobe of lung. Heart mass was measured (Sartorius TE214S Data Weighing Systems Inc Elk Grove, IL, USA), and recorded.

\section{Sample preparation for electron microscopy}

For transmission electron microscopic evaluation of the pulmonary artery, lung tissue and heart tissue samples were fixed with $2.5 \%$ gluteraldehyde, postfixed with $1 \%$ osmium tetroxide, dehydrated in graded alcohol series, cleared with propylene oxide, and embedded in epon. Thin sections (50-70 nm) were cut by a ultramicrotome (Leica UCT-125) and contrasted with uranyl acetate and lead citrate. Sections were examined and photographed under an electron microscope (JEOL JEM-1011).

\section{Statistical methods}

Data were summarized as the mean and standard deviation. Each variant in each group was measured three times and compared using repeated measurements variance analysis. Since initial weigh measurements were found different among groups, so this parameter was accepted as covariant for the model. The differences among groups were identified using the post-hoc Tukey test while, differences in repeated measurements were identified using a contrast method. The differences among groups for heart weigh were evaluated using a covariance analysis. The data were processed and analysed using STATA /MP11. p $<0.05$ ' was accepted as statistically significant.

\section{Results}

Eight of 48 rats in the study died and experimental protocol was completed with 40 rats. None of the rats died treated with sildenafil and SF given during the experiment. Three rats died in each of the combined treatment and bosentan groups and 2 rats in the untreated MCT for various reasons.

Bosentan, sildenafil, SF, and the combination therapy groups were not significantly different in weight gain over time, while the 

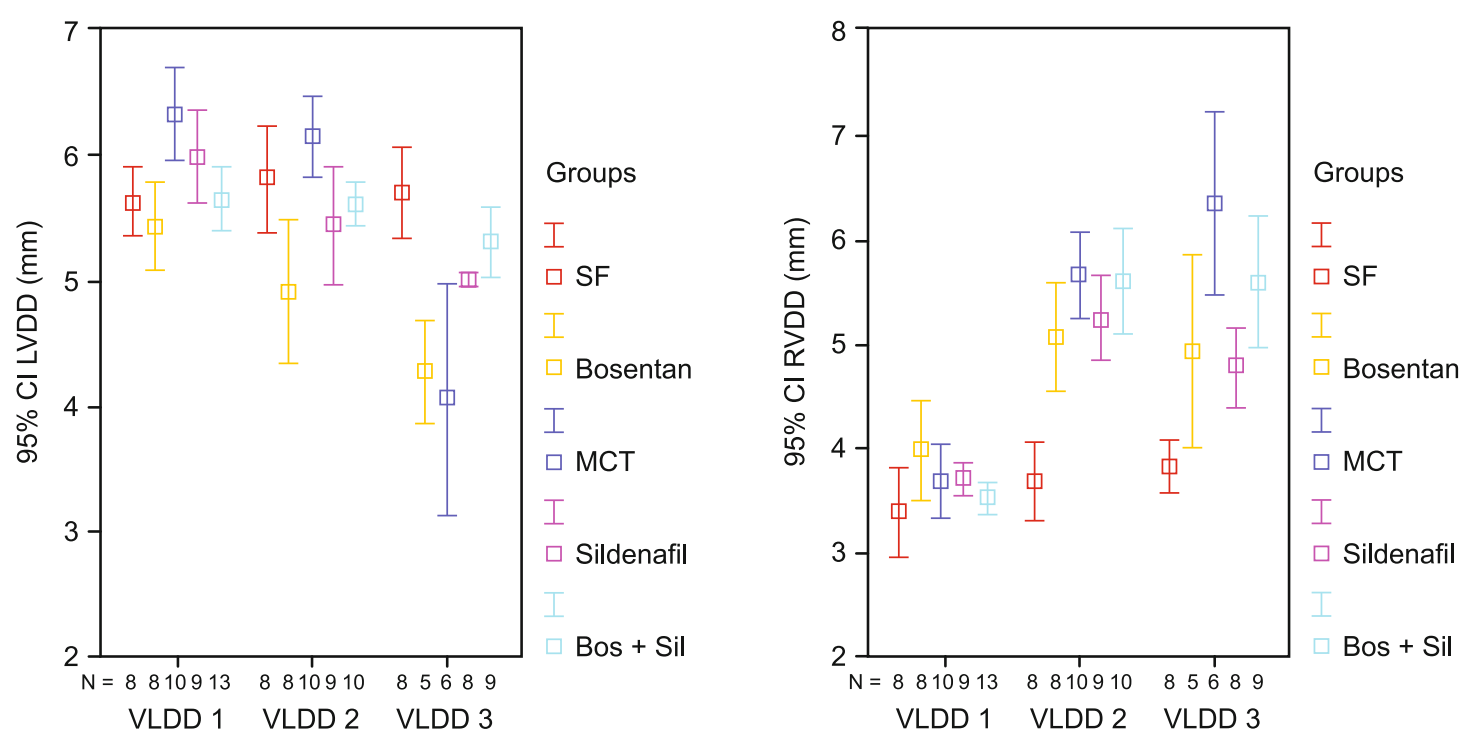

Fig. 1. Mean Values of the Groups for LVDD and RVDD. a: Left ventricular end diastolic diameter (LVDD) values, b: Right ventricular end diastolic diameter (RVDD) values). 1: At the baseline values, 2: Values when PAH developed (4th week), 3: At the end of the treatment (7th week).

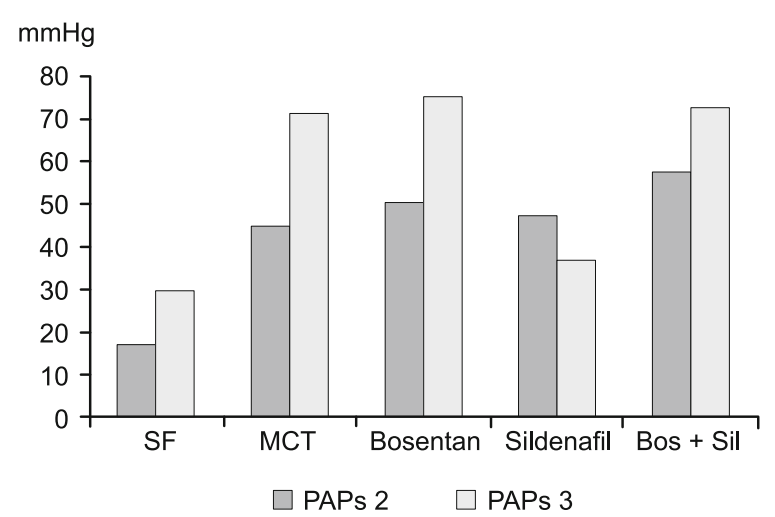

Fig. 2. Echocardiographical Right Ventricle Pressure Change. 1: Pulmonary artery pressure systolic (PAPs), 2: Values when PAH developed (4th week), 3: At the end of the treatment (7th week).

MCT group yes. Significant weight gain was found only in the bosentan therapy group after the development of PH $(\mathrm{p}=0.045)$.

\section{Evaluation of Indirect Blood Pressure Measurement}

Systolic blood pressure and the mean arterial blood pressure values between the groups were obtained both at the beginning and at the end of test with no significant differences.

\section{Evaluation of Echocardiographic Measurements}

Measurements of Left Ventricular End-Diastolic Diameter (LVDD) width in all groups with PH were found significantly lower than in the saline group $(p<0.001)$ (Fig. 1). Diameters of LVDD after the treatment with MCT and bosentan were found lower than in the groups, which received sildenafil and combined therapy.

Significantly increased RVVD values were found in all groups comparing to the saline group when the PH developed $(p<0.001)$

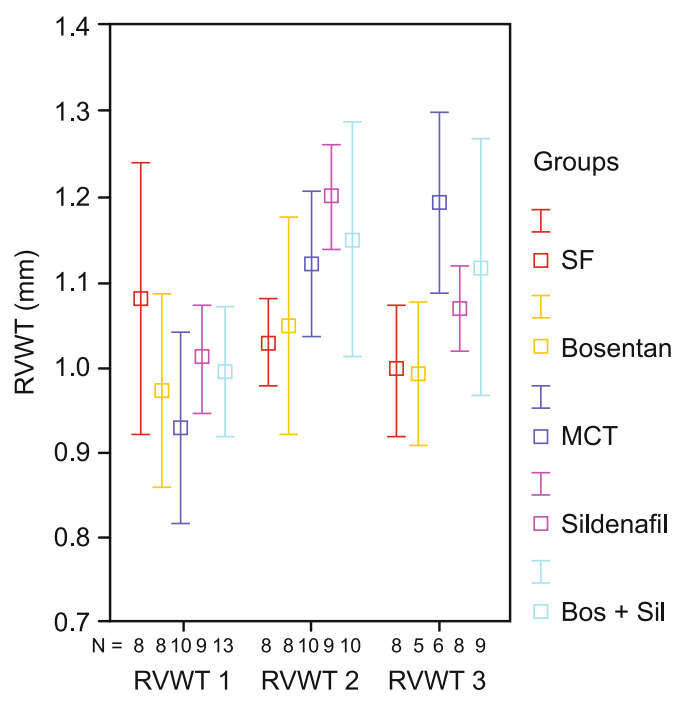

Fig. 3. Average measurement Value on Right Ventricle Wall Thickness (RVWT) of the Groups. Right ventricular (RV. 1: At the baseline values, 2: Values when PAH developed (4th week), 3: At the end of the treatment (7th week).

(Fig. 1). Although there was a slight decrease in the value of RVDD after treatment in all treatment groups, values of RVDD were still significantly wide according to the saline group.

Right ventricular pressure and mean pulmonary artery pressure: At the beginning, there was no significant difference between the groups in right ventricular systolic pressure reflecting the pressure of the PA that was calculated by TY. The second measurements of right ventricular pressure of rats, when the $\mathrm{PH}$ developed, were found significantly increased in comparison with SF group ( $\mathrm{p}<$ 0.001 ). Only right ventricular pressure was significantly reduced 
in sildenafil receiving group after the treatment $(\mathrm{p}<0.001)$ (Fig. 2). Invasive PA and aortic pressure values of the rats were few to take into consideration.

Right ventricular free wall thickness: There were no significant differences among the groups in the measurements of the RVWT at initially. The average RVWT values increased significantly when they had PH and after the treatment $(\mathrm{p}<0.05)$. Only in the third measurement of the sildenafil treatment group, the average of RVWT was significantly thinner (Fig. 3).

Pulmonary artery acceleration time: There was no significant difference in PAAT in between the groups initially. But, there was a significant decrease in PAAT in all groups, when compared to the SF group after the development of PH $(\mathrm{p}<0.001)$. PAAT values were significantly shortened in both groups of treatment and MCT than in the SF group after the treatment. However, PAAT was determined to be longer in the group of treatment than in the MCT group $(\mathrm{p}<0.001)$.

\section{Heart weights}

It was determined that the average heart weight had increased significantly in all the groups compared to the saline group $(\mathrm{p}<$
0.001). The average weight of the heart was the highest in the MCT group whereas it was significantly lower only in the sildenafil group when compared to the MCT group $(\mathrm{p}<0.001)$.

\section{Electron microscopic findings of right ventricle}

The heart muscle cells had normal morphological features in the SF group. Myofibrils and all sarcoplasmic organelles were observed as normal in appearance. Although the heart muscle cells had generally normal morphological features, there were expansions in sarcoplasmic reticulum cisternaes in some heart muscle cells in the other groups (Fig. 4).

\section{Electron microscopic findings of lung}

Alveolar type II epithelial cells had normal morphological features in the SF group. In addition, endothelial cells, alveolar type I epithelial cells and basal lamina of these cells were detected as normal in SF group. Unlike, connective tissue and inflammatory cells were increased in interalveolar septum in other groups. These findings were less dense in the sildenafil group and combined therapy group according to the monocrotaline and bosentan groups (Fig. 5).
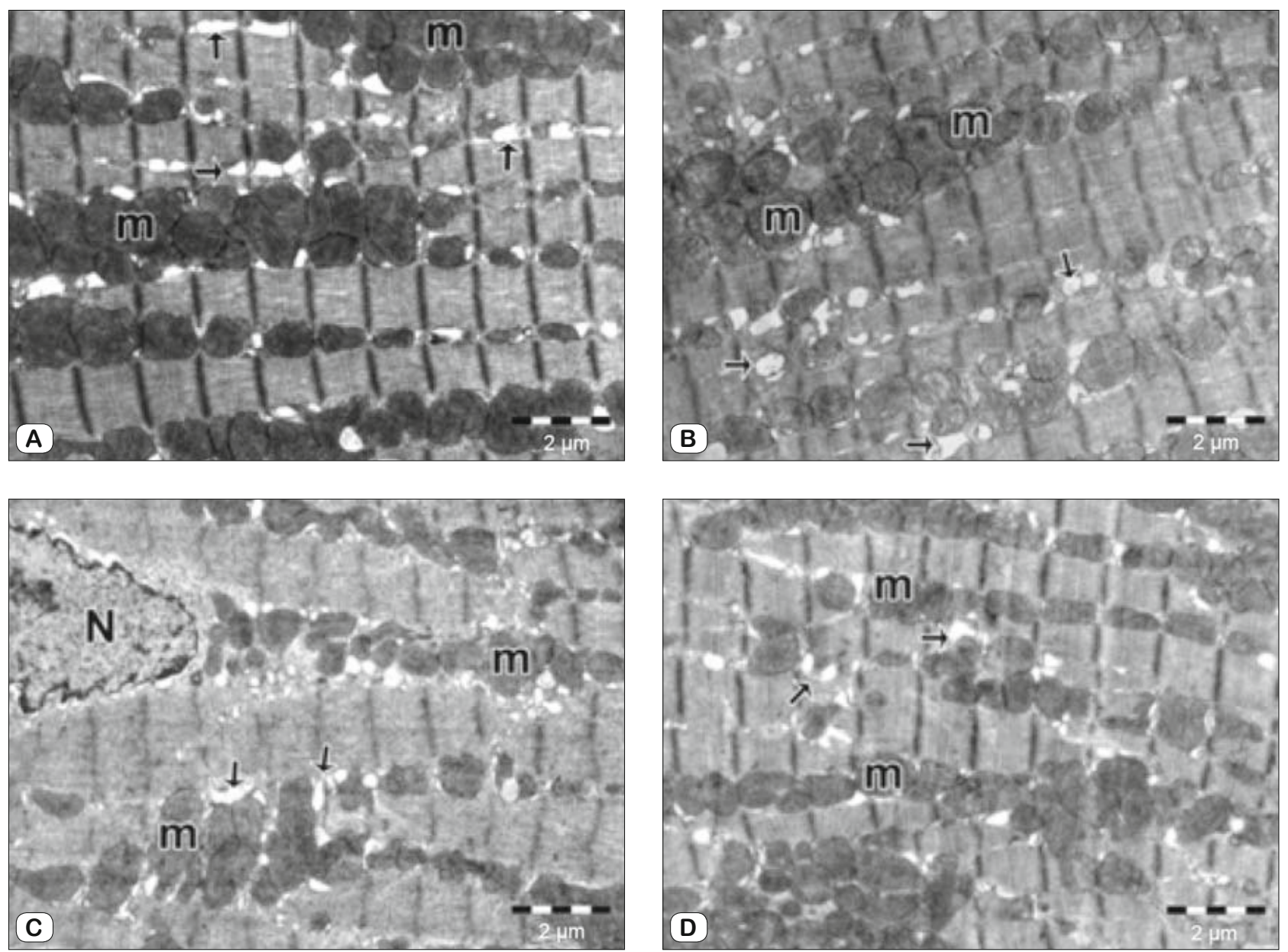

Fig. 4. Electron Microscopic Examination of Right Ventricle of the Groups. A; Monocrotaline (MCT) group, B; MCT+bosentan group, C; MCT+sildenafil group, D; Combined therapy group. Expansions in sarcoplasmic reticulum cisternaes (arrow), mitochondrion (m), nucleus (N). 

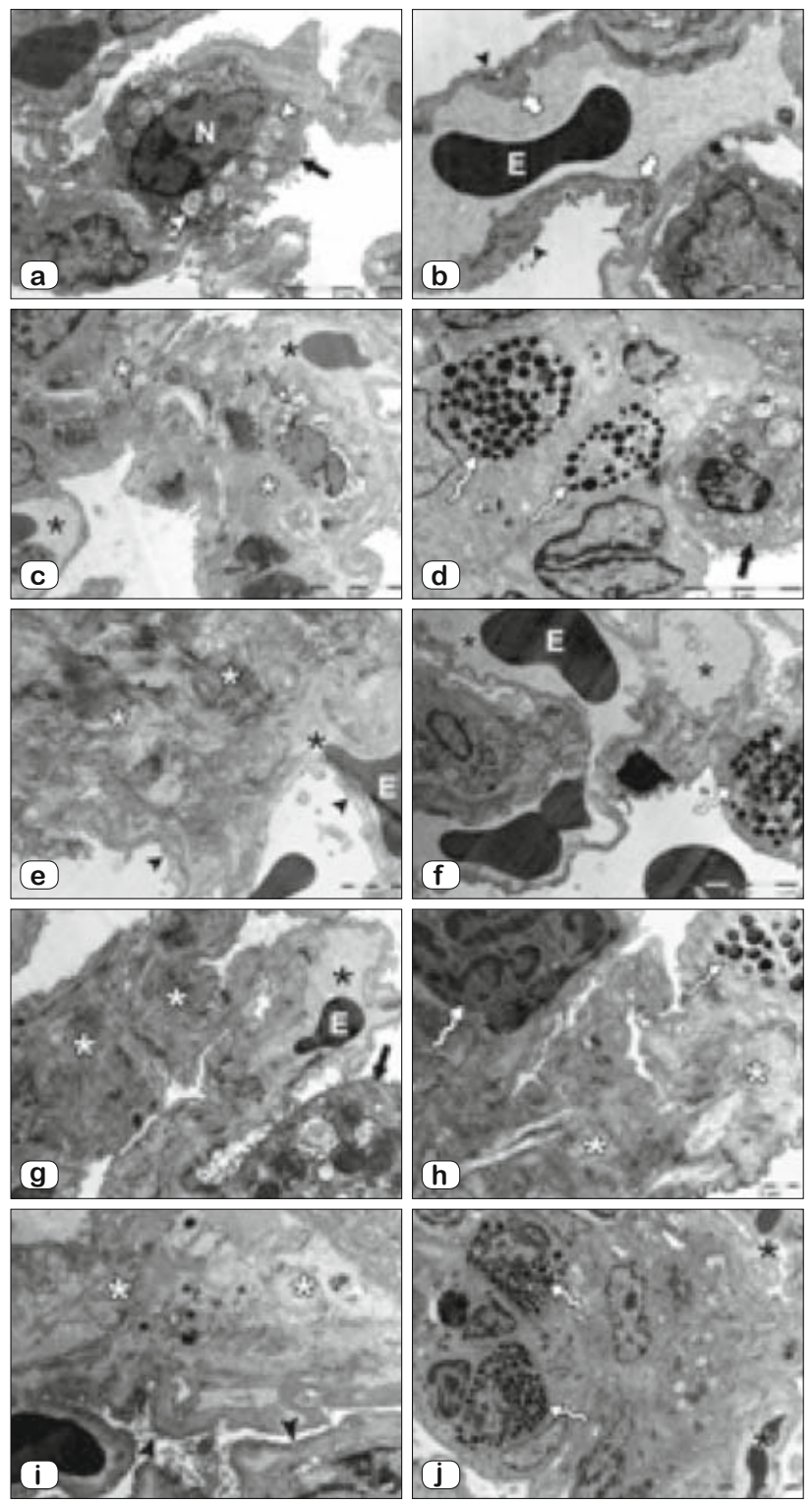

Fig. 5. Electron Microscopic Examination of Lung of the Groups. a, b; Serum physiologic (SF) group, c, d; Monocrotaline (MCT) group, e, f; MCT+bosentan group, g, h; MCT+sildenafil group, i, j; Combined therapy group. Type II pneumocytes (black thick arrow), surfactant vesicles (white arrow head), nucleus (N), erythrocytes (E), type I pneumocytes (black arrow head), endothelial cells (white thick arrow), basal lamina (black thin arrow), connective tissue cells (white thin arrow), connective tissue (white star), blood vessels (black star), inflammatory cells (white curly arrow).

\section{Electron microscopic findings of pulmonary artery}

Ultrastructurally, pulmonary artery had normal morphological features in the control group (group 1). Endothelial cells, smooth muscle cells and connective tissue composition were detected as normal. There were hypertrophic changes and expansions in granular endoplasmic reticulum cisternaes in smooth muscle cells of the group 2, 3, 4 and 5. In addition, the connective tissue volume
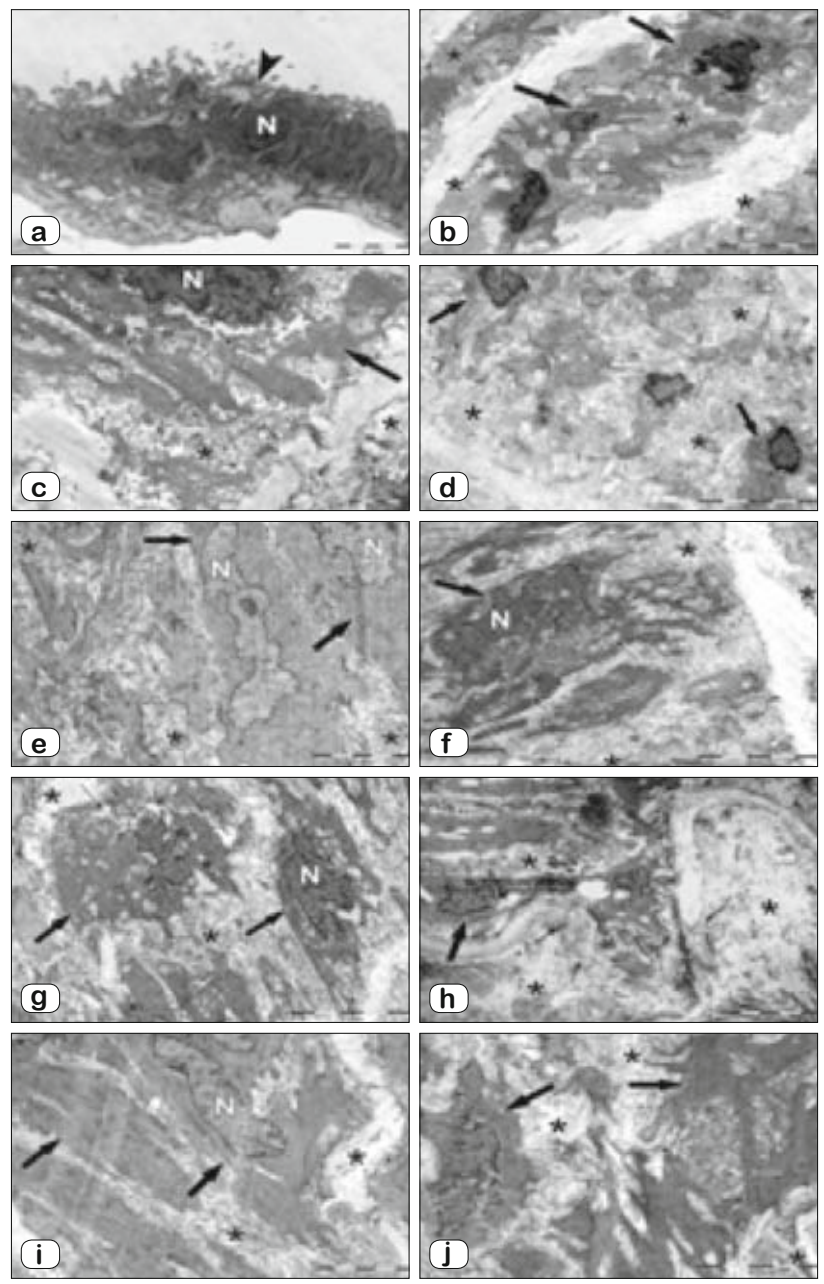

Fig. 6. Electron Microscopic Examination of Pulmonary Artery Cells of the Groups. a, b; Serum physiologic (SF) group, c, d; Monocrotaline (MCT) group, e, f; MCT+bosentan group, g, h; MCT+sildenafil group, i, j; Combined therapy group. Endothelial cell (arrow head), nucleus (N), smooth muscle cell (thick arrow), connective tissue (star), granular endoplasmic reticulum (thin arrow).

was increased in these groups. Ultrastructurally, there were not significantly differences between these groups (Fig. 6).

\section{Discussion}

Sildenafil and the combined therapy were shown to have more apparent effect on right ventricular pressure, right heart and less inflammatory findings on lung tissue histopathologically in rats, in which $\mathrm{PAH}$ was formed experimentally. In addition to this, the histopathologic examination of the right ventricle in all of the groups, where PAH was developed, hypertrophic changes and expansions in sarcoplasmic reticulum cisternaes in smooth muscle cells independent from the applied medication have been revealed. On the other hand, clinical improvement was observed in the group, in which bosentan was administered.

Right ventricular function is the main factor that helps to predict the right ventricular function (14). It is already reported that 
right ventricular hypertrophy and insufficiency develops in PAH that was formed secondary to MCT administration in rats $(15,16)$. Findings of right ventricular insufficiency are weight loss, cyanosis in ears and respiratory difficulty (15). Those changes were observed in all the MCT administered rats in different levels. Two rats died due to right ventricular insufficiency in the MCT group. Weight gain was noted in all the treatment groups and the SF group during the experiment. Weight gain in the bosentan group was statistically significant. Weight gain in the bosentan, sildenafil and combined treatment groups might be accepted as an indicator of clinical improvement.

Many experimental studies have been reported in literature that showed echocardiographic changes in $\mathrm{PH}$ developed in rats following MCT administration (13, 15). Hardziyenka et al has evaluated the relationship between RVWT and right ventricular hypertrophy, RVDD and right ventricular dilatation, and TAPSE and right ventricular contractility in the experimental PAH models. In this study, it has been reported that RVWT and PAAT were the parameters, which were effected first in echocardiography of MCT administered rats (15). The results of our study revealed that PAAT became shorter and increase in RVWT and RVDD have been noted in rats following PAH development. In addition to PAH development, treatment in PAH developed rats was studied in experimental models. Mouchers et al reported that RVDD has had a statistically significant decrease in combined treatment group (17). RVDD was also decreased in sildenafil group without a statistical significance, whereas no decrease at all in bosentan group (17). Clozel et al reported a decrease in right ventricular wall thickness in bosentan, sildenafil and combined therapy groups in PAH formed rats (13). In our study, RVDD decreased in all the treatment groups, especially in the sildenafil group the most, but still there was no statistical significance. RVWT was decreased in all treatment groups; however, the sildenafil group had a statistical significance. Moreover, PAAT became longer in all the treatment groups with respect to MCT group significantly. In rats, in which $\mathrm{PH}$ is developed, right ventricular dilatation occurs whereas LVDD decreases. Mouchers et al has reported that in rats which PAH is formed LVDD was decreased; whereas the values have increased in sildenafil group, but no increase in bosentan group (17). Moreover, similarly, our study also revealed smaller LVDD in the treatment groups when compared to SF group in $\mathrm{PH}$ developed rats. After the completion of therapy, LVDD was increased in sildenafil and combined therapy groups when compared to MCT and bosentan treated groups. Sildenafil increased myocardial hypertrophy by raising cGMP (18). The results obtained in this study showed that sildenafil treatment might be considered as the most effective drug in right ventricular wall thickness.

As it was expected, right ventricular pressure that is measured by non-invasive methods, was higher in $\mathrm{PH}$ developed rats when compared to the SF group. Right ventricular pressure was lower in all treatment groups when compared to MCT group, but statistical significance could only be observed in the sildenafil group. On the basis of this issue, various reports could be found in literature. Right ventricular pressure, which was measured using invasive methods in Wistar rats, did not reveal any statistically significant differences among bosentan, sildenafil and combined therapy groups and no treatment group (17). Clozel et al reported a decrease in PAB levels in all treatment groups; however, this decrease was the clearest in the combined therapy group (13). In a study where experimental PAH was formed, Carlino et al reported a significant decrease in right ventricular pressure when sildenafil and BNP was administered alone; however, when they were administered together, no significant effect was observed (19). Our results showed that sildenafil was more effective on pulmonary pressure when used alone.

Although many mechanisms are described for the pathogenesis of $\mathrm{PH}$, a progressive endothelial cell dysfunction is addressed as the most important one. Factors such as differentiation of vascular smooth muscle cells to endothelial cells and excretion of various mediators or break-up of the balance between mediators play an important role in the pathogenesis of PH (20-23). As a result of this disease, reorganization of pulmonary vessels and, hypertrophy and hyperplasia of the vessel wall occurs $(20,21)$. In all the groups of this study, in which $\mathrm{PH}$ developed, broadening in cisternaes of rough endoplasmic reticulum of smooth muscle cells, hypertrophic findings and increase in volume of connective tissues of pulmonary arteries were noted. Stress in endoplasmic reticulum may occur in many cases, which are related to $\mathrm{PH}$, such as hypoxia and inflammation. As the result of activation of transcription factor 6 , the marker of endoplasmic reticulum stress in pulmonary circulation, mitochondrial dysfunction of endoplasmic reticulum develops. As the result of this, proliferation increases as apoptosis is inhibited (23). In this study, in rats which PAH was developed, broadening of rough endoplasmic reticulum cisternaes, which is responsible for increased protein synthesis, was accounted as a sign for hypertrophy and increase in connective tissue. Histopathologic examination of the right ventricle revealed a broadening of sarcoplasmic reticulum cisternaes in cells located in various regions in all groups other than SF group. Cisternaes of sarcoplasmic reticulum are regions, where calcium is deposited. Intracelular calcium levels are increased in PAH, as the result of a decrease in voltage gates $\mathrm{Kv}$ channels (Kv1.5 and Kv4.2), which are responsible for repolarization in right ventricular myocytes $(24,25)$. Broadening of cisternaes of rough sarcoplasmic reticulum is observed in rats, in which PAH is developed, and this may be due to the increase in calcium depositions in order to increase cardiac contractility.

Vascular damage occurs in PAH as the result of increase in cytokins and growth factors, which in turn causes an invasion of lung tissue with monocytes and macrophages. Electron microscope examination of lung tissue revealed a significance of connective tissue and increase in inflammatory cells were also observed in all the groups except the SF group. Abundance of inflammatory cells were lower in the sildenafil and the combined therapy groups. Thus, sildenafil and the combined therapy groups showed a better improvement histopathologically in lung tissue when compared to the other groups.

A progressive constriction of pulmonary arteries lie in the pathogenesis of $\mathrm{PH}$ and this commonly result in a right sided heart failure and death $(26,27)$. Right ventricle dysfunction is the most important predictor of the life expectancy in patients with PAH 
(14-16). Although it is not exactly known yet, sildenafil's effect on right ventricular insufficiency in PAH treatment might possibly be either through pulmonary vascularity or through direct antihypertrophic effect of right ventricular remodelling. In the study by Xie et al sildenafil treatment was given to rats, in which PAH was developed following MCT administration, and the effects of sildenafil on right ventricular insufficiency was evaluated. It was concluded that early sildenafil treatment prevents right ventricular hypertrophy and insufficiency, T tubular remodelling and, calcium retention dysfunction (28).

PAH is a life- threatening disease with bad prognosis and shows a rapid progression. Early diagnosis and treatment has a positive impact on clinical and hemodynamic changes in long term. Combination therapy, which targets different pathological processes became the treatment of choice. However, many questions such as decision-making, on which drug to use, timing, and the effectivity of these drugs when used simultaneously, stay still unanswered. It has been known that pharmacokinetic interaction occurs when bosentan and sildenafil are used simultaneously. When bosentan and sildenafil are used as a combined therapy, bosentan decreases sildenafil levels by increasing activities of CYP3A4 and CYP2C9, which are known as cytochrome P450 isoenzymes. However, sildenafil increases bosentan levels by inhibiting hepatic uptake of OATP1B1 and OATPB3, which are known as organic anion transport polypeptides (29). So far, no findings were reported to prove a decrease in security due to this interaction (30). However, it is still controversial, whether the clinical efficacy of sildenafil decreases when used as a combined therapy with bosentan. In the study, which was performed in healthy volunteers within whom the approved dosages of combined therapy of bosentan and sildenafil was used, bosentan levels exceeded maximum concentration by 42 $\%$, while sildenafil levels lowered the maximum concentration by $55 \%$. Those reports showed that pharmacokinetic interaction between bosentan and sildenafil must be investigated in more details.

In conclusion, this experimental study showed that sildenafil and the combined therapy were more effective in rats with PAH. Although echocardiography and hemodynamic results did not show an apparent improvement, clinical recovery was considered. The main drawback of this study is that experimental models and clinical models may not be comparable exactly. However, studies on human subjects may provide more reliable data. Since histopathological studies may not be performed in human subjects due to ethical limitations, some studies must be performed experimentally. Although the use of sildenafil and bosentan deems suitable as the result of the clinical and experimental studies performed so far, it is not possible to decide whether the individual effectivity for each of the drugs is different when used alone or in combination. In spite of the partial improvement in survival and hemodynamic findings with the use of the present treatment options in $\mathrm{PAH}$, death rates still remain high. Due to this reason, further studies targeting various biopathological mechanisms, which will result in better clinical outcome and prognosis, must be performed.

\section{References}

1. D’Alonzo GE, Barst RJ, Ayres SM et al. Survival in patients with primary pulmonary hypertension. Results from a national prospective registr. Ann Intern Med 1991; 115: 343-349.

2. Gaine SP, Rubin LJ. Primary pulmonary hypertension. Lancet 1998; 352: 719-725.

3. Rich S, Dantzker DR, Ayres SM et al. Primary pulmonary hypertension. A national prospective study. Ann Intern Med 1987; 107: 216-223.

4. Kalogeropoulos AP, Vega D, Smith AL, Georgiopoulou VV. Pulmonary hypertension and right ventricular function in advanced heart failure. Congest Heart Fail 2011; 17: 189-198.

5. Bogard HJ, Natarajan R, Henderson SC et al. Chronic pulmonary artery pressure elevation is insufficient to explain right heart failure. Circulation 2009 ; 17 ; 120: 1951-1960.

6. Galie` N, Rubin LJ, Hoeper M et al. Treatment of patients with mildly symptomatic pulmonary arterial hypertension with bosentan (EARLY study): a double-blind, randomised controlled trial. Lancet 2008; 371 (8): 2093-2100.

7. Rubin LJ, Badesch DB. Evaluation and management of the patient with pulmonary arterial hypertension. Ann Intern Med 2005; 143: 282-292.

8. Rubin LJ, Badesch DB, Barst RJ et al. Bosentan therapy for pulmonary arterial hypertension. N Engl J Med 2002; 346: 896-903.

9. Channick RN, Simonneau G, Sitbon O et al. Effects of the dual endothelin-receptor antagonist bosentan in patients with pulmonary hypertension: a randomised placebo-controlled study. Lancet 2001; 358: 1119-1123.

10. Galie N, Ghofrani HA, Torbicki A et al. The Sildenafil Use in Pulmonary Arterial Hypertension (SUPER) Study Group. Sildenafil citrate therapy for pulmonary arterial hypertension. New Engl J Med 2005; 353: 2148-2157.

11. Stenmark KR, Meyrick B, Galie N, McMurty IF. Animal models of pulmonary arterial hypertension: the hope for etiolological discovery and pharmacological cure. Am J Physiol 2009; 297: 1013-1032.

12. Dias-Neto M, Luísa-Neves A, Pinho S et al. Pathophysiology of Infantile Pulmonary Arterial Hypertension Induced by Monocrotaline. Pediatr Cardiol 2015; 36: 1000-1013.

13. Clozel M, Hess P, Rey M, Iglarz M. Bosentan, Sildenafil, and Their Combination in the Monocrotaline Model of Pulmonary Hypertension in Rats. Exp Biol Med 2006; 231: 967-973.

14. Van Oort RJ, Garbino A, Wang W, Dixit SS et al. Disrupted junctional membrane complexes and hyperactive ryanodine receptors after acute junctophilin knockdown in mice. Circulation 2011; 123: 979 -988.

15. Hardziyenka M, Campian ME, Bruin-Bon HA, Michel MC. Sequence of echocardiographic changes during development of right ventricular failure in rat. J Am Soc Echocardiogr 2006; 19: 1272-1279.

16. Jones JE, Mendes L, Rudd MA, Russo G, Loscalzo J, Zhang YY. Serial noninvasive assessment of progressive pulmonary hypertension a rat model. Am J Physiol 2002; 283: H364-371.

17. Mouchaers KT, Schalij I, Versteilen AM et al. Endothelin receptor blockade combined with phosphodiesterase-5 inhibition increases right ventricular mitochondrial capacity in pulmonary hypertension. Am J Physiol Heart Circ Physiol 2009; 297: 200-207.

18. Hassan MA, Ketat F. Sildenafil citrate increases myocardial cGMP content in rat heart, decreases its hypertrophic response to isoproterenol and decreases myocardial leak of creatine kinase and troponin T. BMC Pharmacol 2005; 5-10. 
19. Carlino C, Tobias JD, Schneider R et al. Pulmonary hemodynamic response to acute combination and monotherapy with sildenafil and brain natriuretic peptide in rats with monocrotaline-induced pulmonary hypertension. Am J Med Sci 2010; 339: 55-59.

20. Humbert M, Sitbon O, Simoneau G. Treatment of Pulmonary Arterial Hypertension. N Engl J Med 2004; 351: 1425-1436.

21. Humbert M, Morrell NW, Archer SL et al. Cellular and molecular pathobiology of pulmonary arterial hypertension. J Am Coll Cardiol 2004; 43: 13-24.

22. Chan SY, Loscalzo J. Pathogenic mechanisms of pulmonary arterial hypertension. J Mol Cell Cardiol 2008; 44: 14-30.

23. Dromparis P, Paulin R, Stenson TH, Haromy A, Sutendra G, Michelakis ED. Attenuating endoplasmic reticulum stress as a novel therapeutic strategy in pulmonary hypertension. Circulation 2013; 127 (1): 115-125.

24. Piao L, Fang YH, Cadete VJ et al. The inhibition of pyruvate dehydrogenase kinase improves impaired cardiac function and electrical remodeling in two models of right ventricular hypertrophy: resuscitating the hibernating right ventricle. J Mol Med 2010; 88: 47-60.

25. Benoist D, Stones R, Drinkhill MJ et al. Cardiac arrhythmia mechanisms in rats with heart failure induced by pulmonary hypertension. Am J Physiol Heart Circ Physiol 2012; 302: 2381-2395.
26. Humbert M, Sitbon O, Chaouat A et al. Pulmonary arterial hypertension in France: results from a national registry. Am J Resp Crit Care Med 2006; 173: 1023-1030.

27. Oka M, Homma N, Taraseviciene-Stewart L et al. Rho kinase-mediated vasoconstriction is important in severe occlusive pulmonary arterial hypertension in rats. Circ Res 2007; 100: 923-929.

28. Xie YP, Chen B, Sanders $P$ et al. Sildenafil Prevents and Reverses Transverse-Tubule Remodeling and Ca2 Handling Dysfunction in Right Ventricle Failure Induced by Pulmonary Artery Hypertension. Hypertension 2012; 59 (2): 355-362.

29. Treiber A, Schneiter R, Hausler S, Stieger B. Bosentan is a substrate of human OATP1B1 and OATP1B3: inhibition of hepatic uptake as the common mechanism of its interactions with cyclosporine $\mathrm{A}$, rifampicin, and sildenafil. Drug Metab Dispos 2007; 35: 1400-1407.

30. Humbert M, Segal ES, Kiely DG, Carlsen J, Schwierin B, Hoeper MM. Results of Europen post-marketing surveillance of bosentan in pulmonary hypertension. Eur Resp J 2007; 30: 338-344.

31. Burgess G, Hoogkamer H, Collings L, Dingemanse J. Mutual pharmacokinetic interactions between steady-state bosentan and sildenafil. Eur J Clin Pharmacol 2008; 64: 43-50. 